\title{
Valuation and Risk Assessment of a Portfolio of Variable Annuities: A Vector Autoregression Approach
}

\author{
Albina Orlandoํ, Gary Parker ${ }^{2}$ \\ ${ }^{1}$ Istituto per le Applicazioni del Calcolo "Mauro Picone" CNR, Napoli, Italy \\ ${ }^{2}$ Department of Statistics and Actuarial Science, Simon Fraser University, Burnaby, Canada \\ Email: a.orlando@na.iac.cnr.it, gparker@stat.sfu.ca
}

How to cite this paper: Orlando, A. and Parker, G. (2018) Valuation and Risk Assessment of a Portfolio of Variable Annuities: A Vector Autoregression Approach. Journal of Mathematical Finance, 8, 349-371. https://doi.org/10.4236/jmf.2018.82023

Received: February 11, 2018

Accepted: May 7, 2018

Published: May 10, 2018

Copyright (c) 2018 by authors and Scientific Research Publishing Inc. This work is licensed under the Creative Commons Attribution International License (CC BY 4.0).

http://creativecommons.org/licenses/by/4.0/

\section{c) (i) Open Access}

\begin{abstract}
This paper focuses on assessing the financial position of an insurer issuing a portfolio of Variable Annuities (VAs). Two multivariate models for the underlying and the interest rate are considered. The first model uses a single total rate of return for the basket of assets. The second one, jointly models the rates of return on the $\mathrm{n}$ assets in the basket. For simplicity, the insurer is assumed to be able to implement a static hedging programme to manage the risk. As an example, a homogeneous portfolio of VAs with GMDB and GMMB guarantees offering different investment opportunities to the policyholders is studied. The insurer can choose to rebalance the basket of assets regularly or not. Results for these two cases are presented.
\end{abstract}

\section{Keywords}

Variable Annuities, VAR Models, Static Hedging, Conditional Value at Risk

\section{Introduction}

Over the years, many practical and academic contributions have been offered describing VAs and their embedded guarantees.

Variable annuities (VAs) are life insurance products with investment guarantees providing substantial investment guarantees along with tax privileges and prudently managed assets. VAs have existed in USA since the 1950s. Recently they have been spreading across Europe. Some of the more significant and high profile launches have been AXA in France, Germany, Spain, Italy and Belgium as well as ING launches in Spain, Hungary and Poland, Generali launch (December 2007) in Italy and Ergo launch (February 2008) in Germany. This is 
in addition to the various launches by Aegon, Hartford, Metlife and Lincoln in the U.K. [1].

VAs allow the allocation of premiums into a range of investment options which usually contain stocks, bonds, money market instruments or some combination of the three. The benefits to the policyholder will depend on the performance of the chosen investment option; typically, the benefit is the higher of the account value and the guaranteed amount.

The major types of VA guarantees are: Guaranteed Minimum Death Benefits (GMDBs) that guarantee a return of the principal invested upon the death of the policyholder; Guaranteed Minimum Accumulation Benefits (GMABs) where the guarantees typically bite on specified policy anniversaries or between specified dates if the policy is still in force. If guarantees are available at maturity they are called Guaranteed Minimum Maturity Benefits (GMMBs); Guaranteed Income Benefits (GMIBs) guarantee a minimum income stream (typically in the form of a life annuity) from a specified future point in time; Guaranteed Minimum Withdrawal Benefits (GMWBs) guarantee a minimum income stream through regular withdrawals from the account.

A detailed overview of variable annuities and a description of the VA international market are given in [1] and [2]. The main emphasis of the current literature is on pricing and hedging the options embedded in these life insurance contracts. A general framework in which any design of options and guarantees currently offered within VAs can be modeled, is presented in [3]. In the recent literature we find some interesting contributions on identification and assessment of risk. Among others, [4] deals with risk assessment, mostly via stochastic simulation. Their main contribution is to present an integrated approach to risks in VA products. Analytical methods for the calculation of risk measures for variable annuities guaranteed benefits and a quantitative model for market risk on the liability side and revenue risk in the asset side, are proposed in [5].

The insurer will be holding the liability for the VAs guarantee until lapse, death or maturity. The impact of both mortality risks (due to random fluctuations in the number of deaths) and investment risks can be rather high in the presence of some types of guarantees and options. The resulting economic impact to the insurer selling the guarantee is based on how the future real world scenario plays out. Given these considerations, it may be useful for an insurer to assess real world risk associated with embedded guarantees of its VA portfolio, once pricing is done and a risk management strategy is adopted.

Most insurers manage the risk of their VA portfolios by adopting a sophisticated hedging programme. Some use static hedging to mitigate the risk, at least partially. Here, static hedging refers to strategies consisting of buying and selling long-term financial instruments, e.g. over the counter (OTC) options, at time of issue and holding them until maturity with little rebalancing, if any. Available static hedges often fail to closely match the liabilities of VA products 
and are expensive to set up. For these reasons, dynamic hedging strategies are more popular among insurers. Here, a portfolio of liquid assets is set up so that, over a short period, its change in value will approximately match the change in value of the liabilities. The portfolio of assets is then rebalanced at a frequency that takes into account trading costs and effectiveness of the hedge.

In this paper, it is assumed that the insurer is able to implement a static hedge. This allows us to compare the impact of the two multivariate processes used to model the rate of return of the basket of assets on the value of the portfolio. We can also study whether rebalancing the basket of assets held in the VA account or not affects the risk. Although using a dynamic hedging strategy is more realistic, we consider a static hedging program as a first step of our research which is not specifically focused on the kind of strategy chosen to hedge the portfolio. Dynamic hedging will be explored in future work.

In this paper we choose to focus on assessing the financial position at issue of an insurer selling a portfolio of VA products offering GMDB and GMMB guarantees. We measure the portfolio value at inception as the difference between the present values of the stochastic future inflows and outflows in a real world scenario.

Our main contribution consists of considering two multivariate VAR (1) processes to model the underlying and the interest rate and compare the portfolio values at inception.

Although variable annuities are written on more than one asset, in practice, single asset univariate stochastic investment models are mostly used for pricing and hedging purposes. This may lead to problems such as basis risk or a lack of flexibility with respect to asset allocations [6]. In light of these considerations, we compare a two-dimensional VAR (1) model, where the asset returns on each fund are combined in a single time series, to an $(n+1)$-dimensional VAR (1) model where the asset returns on each fund are modelled separately. A special case of these models is proposed to better capture the mutual dependence of the variables involved.

Another issue for the insurer is the decision to rebalance or not the basket of assets constituting the fund itself. Rebalancing is the process of realigning the weightings of a portfolio of assets. It involves periodically buying and selling assets in a portfolio to maintain a specific asset allocation. In our work, we consider two different accumulation functions depending on whether the basket of assets is rebalanced or not. For sake of simplicity, no transaction costs are considered.

Finally, we assume that the VA writer offers to policyholders different premium allocations with different investment styles. These kind of Funds characterize some VA products sold in Europe (among others, the "Accumulator" sold by AXA).

The different cases described above are evaluated in a simulation framework. The results under static hedging are compared with those obtained when the 
insurer decides not to hedge.

The remainder of the paper is organized as follows. Section 2 introduces the product, Section 3 describes the VAR (1) models employed, Section 4 is devoted to the portfolio evaluation at inception. In Section 5, the results are presented and discussed. In Section 6, some concluding remarks are given.

\section{The Contract}

Let us consider a portfolio of VA contracts offering GMDB and GMMB guarantees issued to $C$ independent lives aged $x$, each paying a unique premium $P$ at issue.

The insurer invests the premiums, net of initial charges for general expenses, into several internal funds characterized by different investment styles.

Policyholders may choose in which funds to invest.

By virtue of the GMDB guarantee, if the insured dies in policy year $t$, the insurer pays a sum equal to the greater of the guaranteed amount or the fund value.

On the other hand, by virtue of the GMMB, at maturity $T$ the insurer pays the greater of the guaranteed amount or the fund value, if the insured is still alive. The benefits are paid monthly. A monthly management charge is deducted and the money gathered by the insurer through this charge is used to cover the guarantees and other expenses linked to the Fund such as fund management and taxes.

The obligations the insurer has to front for the GMDB at time $t$ are valued

$$
G M D B_{t}=N_{D}(t) \cdot \operatorname{Max}\left[F_{t}, G_{t}\right]
$$

with $t \in[1,2, \cdots, T]$.

For the GMMB we have:

$$
G M M B_{T}=N_{S}(T) \cdot \operatorname{Max}\left[F_{T}, G_{T}\right]
$$

where $N_{D}(t)$ is the actual number of deaths in $[t-1, t]$ and $N_{S}(T)$ is the actual number of survivors at time $T$.

According to the contractual design, the fund value used in (1) and (2) is:

$$
F_{t}=P^{\prime} \cdot(1-m)^{t} e^{f(t)}
$$

and the guaranteed value is:

$$
G_{t}=P^{\prime} \cdot e^{g \cdot t}
$$

with $t \in[1,2, \cdots, T]$.

Here, $F_{t}$ is the fund value obtained by investing the net premiums $P^{\prime}=P-c$ into the chosen internal funds, $c$ is the initial charge and $P$ the single premium. $e^{f(t)}$ is the accumulation function and $m$ the monthly management charge deducted. $G_{t}$ is the guaranteed amount obtained by accumulating the net premium at the guaranteed rate $g$ (roll up).

Distinct funds are offered, each made up of a mix of several assets. The combination of the assets gives rise to various investment styles. 
Moreover, with reference to the fund value $F_{t}$ in (3), we consider two cases: the rebalancing strategy of the basket of assets constituting the fund itself and the no rebalancing one (see Section 3).

By means of the well known put decomposition principle, we can rewrite the guarantees (1) and (2) as follows:

$$
G M D B_{t}=N_{D}(t) \cdot\left(F_{t}+\left[G_{t}-F_{t}, 0\right]_{+}\right)
$$

with $t \in[1,2, \cdots, T]$ and

$$
G M M B_{T}=N_{S}(T) \cdot\left(F_{T}+\left[G_{T}-F_{T}, 0\right]_{+}\right) .
$$

(5) and (6) imply that upon the death of the policyholder or expiration of the contract, the insurance Company pays the accumulated fund value $F_{t}$ to the investor plus an additional payoff of a put option with strike price equal to $G_{t}$ with $t \in[1,2, \cdots, T]$ and $G_{T}$ respectively.

Therefore if the fund performs so poorly that its value, when the contract matures or when death occurs, is below the corresponding guaranteed values, the insurer pays the difference.

For the GMDB, the insurer's monthly payments are:

$$
L_{t}^{G M D B}=N_{D}(t) \cdot\left[G_{t}-F_{t}, 0\right]_{+} .
$$

with $t \in[1,2, \cdots, T]$.

On the other hand for the GMMB we have:

$$
L_{T}^{G M M B}=N_{S}(T) \cdot\left[G_{T}-F_{T}, 0\right]_{+}
$$

The random liabilities evaluated at time zero are then given by:

$$
\begin{aligned}
& L_{0}^{G M D B}=\sum_{t=1}^{T} N_{D}(t) \cdot\left(G_{t}-F_{t}, 0\right)_{+} \cdot e^{-y(t)} \\
& L_{0}^{G M M B}=N_{S}(t) \cdot\left(G_{T}-F_{T}, 0\right)_{+} \cdot e^{-y(T)}
\end{aligned}
$$

where $e^{-y(t)} \quad t \in[1,2, \cdots, T]$ is a random discounting factor.

\section{Modeling the Financial Variables: The Vector Autoregressive Model}

We choose a multivariate framework modelling jointly all financial variables, namely asset returns and interest rates, by means of a Vector Autoregressive model of order one, VAR (1).

The VAR model is one of the most flexible and easy to use models for analyzing multivariate time series. It is a natural extension of the univariate autoregressive model to study multivariate time series. The VAR model has proven to be especially useful for describing the dynamic behaviour of economic and financial time series and for forecasting [7].

An n-dimensional time series random variable $X_{s}$ is said to follow a VAR (1) model if 


$$
\underline{X_{s}}-\underline{\mu}=\underline{\Phi} \cdot\left(\underline{X_{s-1}}-\underline{\mu}\right)+\underline{a_{s}}
$$

where: $s \in Z, \underline{\mu}$ is an $(n \times 1)$ matrix of the long term mean values of $X_{s}$, $\underline{\Phi}$ is an $(n \times n)$ matrix of autoregressive coefficients, $a_{s}$ is an n-dimensional white noise term which follows a multivariate normal distribution with zero mean and covariance matrix $\sum_{a}$.

A sufficient condition of stationarity is that all eigenvalues of $\underline{\Phi}$ are less than 1 in absolute value.

When conditioning on the initial value $\underline{X_{0}}$, the mean of $\underline{X_{s}}$ is

$$
\left.E\left[\underline{X_{s}} \mid \underline{X_{0}}\right]=\underline{\Phi^{s}} \cdot \underline{\left(\underline{X_{0}}\right.}-\underline{\mu}\right)+\underline{\mu}
$$

The covariance between $\underline{X_{s}}$ and $\underline{X_{s-k}}$, where $k$ is a non negative integer, $k<s$, also conditional on $\underline{X_{0}}$ is:

$$
\operatorname{Cov}\left[\underline{X_{s}}, \underline{X_{s-k}} \mid \underline{X_{0}}\right]=\sum_{i=0}^{s-k-1} \underline{\Phi^{s+i}} \cdot \underline{\Sigma}_{a} \cdot \underline{\Phi}^{i}
$$

We consider two models: a two-dimensional VAR (1) model for the composite rate of return of the $n$ assets and the interest rate and a $(n+1)$-dimensional VAR (1) for the rates of return and the interest rate.

\subsection{Two-Dimensional VAR (1) Model}

In this case we model the composite rate of return $\delta_{s}$ of the $n$ assets constituting each fund and the interest rate $r_{s}$ by means of a two-dimensional VAR (1) model.

Referring to (11) we can write:

$$
\begin{gathered}
\underline{X_{s}}=\left(\begin{array}{l}
\delta_{s} \\
r_{s}
\end{array}\right) \\
\underline{\Phi}=\left(\begin{array}{ll}
\Phi_{1,1} & \Phi_{1,2} \\
\Phi_{2,1} & \Phi_{2,2}
\end{array}\right) \\
\underline{a_{s}}=\left(\begin{array}{l}
a_{\delta_{s}} \\
a_{r_{s}}
\end{array}\right)
\end{gathered}
$$

The composite rate of return $\delta_{s}$ can be computed in two ways, depending on whether the basket of assets is rebalanced or not. When rebalancing the basket of assets we have:

$$
\delta_{s}=\sum_{i=1}^{n} \omega_{i} \cdot \delta_{s}^{i}
$$

with no rebalancing, the composite rate of return is:

$$
\delta_{s}=\sum_{i=1}^{n}\left(\prod_{k=1}^{s-1}\left(1+\delta_{s-k}^{i}\right) * \delta_{s}^{i}\right)
$$

where $\delta_{1}=\sum_{i=1}^{n} \omega_{i} \cdot \delta_{1}^{i}$ and $t \in 1,2, \cdots, T$.

In (17) and (18) $\delta_{s}^{i}$ with $i \in 1,2, \cdots, n$ are the monthly continuous rates of return of the $n$ assets and $\omega_{i} \quad i \in 1,2, \cdots, n$ are the proportion of the fund 
invested in each of the $n$ assets.

If we model the financial variables by a two-dimensional VAR (1) model, $F_{t}$ in (3) can be rewritten as:

$$
F_{t}=P^{\prime} \cdot(1-m)^{t} \cdot e^{\sum_{s=1}^{t} \delta_{s}}
$$

with $t \in[1,2, \cdots, T]$.

In (19), $\delta_{s}$ is given by (17) or (18) depending on whether the fund is rebalanced or not. $F_{t}$ assumes different values depending on the selected investment strategy.

The discounting factor is given by:

$$
e^{-y(t)}=e^{-\sum_{s=1}^{t} r_{s}}
$$

with $t \in[1,2, \cdots, T]$.

\section{2. $(n+1)$-Dimensional VAR (1) Model}

Here we consider a $(n+1)$-dimensional VAR $(1)$, modelling jointly the rates of return of the $n$ assets in which the insurer invests $\left(\delta_{s}^{i}\right.$ with $\left.i \in 1,2, \cdots, n\right)$ and the discounting interest rate $\left(r_{s}\right)$.

Referring to (11) we can write:

$$
\begin{aligned}
& \underline{X_{s}}=\left(\delta_{s}^{1}, \delta_{s}^{2}, \delta_{s}^{3}, \cdots, \delta_{s}^{n}, r_{s}\right)^{\mathrm{T}} \\
& \underline{\Phi}=\left(\begin{array}{ccccc}
\Phi_{1,1} & \Phi_{1,2} & \cdots & \Phi_{1, n} & \Phi_{1, n+1} \\
\Phi_{2,1} & \Phi_{2,2} & \cdots & \Phi_{2, n} & \Phi_{2, n+1} \\
\vdots & \vdots & \ddots & \vdots & \vdots \\
\Phi_{n, 1} & \Phi_{n, 2} & \cdots & \Phi_{n, n} & \Phi_{n, n+1} \\
\Phi_{n+1,1} & \Phi_{n+1,2} & \cdots & \Phi_{n+1, n} & \Phi_{n+1, n+1}
\end{array}\right) \\
& \underline{a_{s}}=\left(a_{\delta_{s}^{1}}, a_{\delta_{s}^{2}}, a_{\delta_{s}^{3}}, \cdots, a_{\delta_{s}^{n}}, a_{r_{s}}\right)^{\mathrm{T}} .
\end{aligned}
$$

Based on some observations on the estimated parameters, we also propose a modified version of the $(n+1)$-VAR (1) which includes a Geometric Brownian Motion (GBM) to model the stock indexes (see Section 5).

The use of the $(n+1)$-dimensional model implies that we have different values of $F_{t}$ in (3), in case of rebalancing or not the basket of assets

1) Rebalancing case:

$$
F_{t}=P^{\prime} \cdot(1-m)^{t} \cdot e^{\sum_{s=1}^{t} \sum_{i=1}^{n} \omega_{i} \cdot \delta_{s}^{i}}
$$

2) No rebalancing case:

$$
F_{t}=\sum_{i=1}^{n} \omega_{i} \cdot P^{\prime} \cdot(1-m)^{t} \cdot e^{\sum_{s=1}^{t} \delta_{s}^{i}}
$$

where $\varpi_{i} \quad i \in[1,2, \cdots, n]$ are the percentages of capital invested by the insurer in each of the $n$ assets.

In both cases, according to the values of $\omega_{i}$, we obtain different accumulation factors depending on the chosen investment style.

The discounting factor is given by: 


$$
e^{-y(t)}=e^{-\sum_{s=1}^{t} r_{s}}
$$

with $t \in[1,2, \cdots, T]$

\section{Hedging the Guarantee}

Bearing in mind that the VA contracts embed equity put options, a crucial problem is to hedge these options.

Hedging the put options means holding a hedge portfolio replicating the guarantee payoff. A possible solution is dynamic hedging which requires a continuous rebalancing of the hedge portfolio.

In this framework, we consider the case of static hedging; for example, the insurer buys equity puts on the market place.

\subsection{The Cost of the Hedging Portfolio}

In case of static hedging, the insurer buys a portfolio of put options, at time zero, to hedge the guarantee payoff. We know that, in this case, the option price may be too high because these options differ in nature and term from the typical over the counter options currently available. Moreover, the counter party risk may be a factor, given the long terms involved.

Static hedging is not always possible, neverthless this is a first step of our study. Future research will consider dynamic hedging, too.

We price the replicating portfolio by adapting the Black-Scholes results for the guarantees embedded in the VA contract like in [8] while considering the different assumptions we make about the financial variables.

The term to maturity of each option in the VA contract is random, depending on the remaining lifetime of the policyholders. For the GMDB guarantee it depends on the monthly death probabilities and for the GMMB it depends on the survival probability at the maturity of the contract.

Therefore, the expected cost of the hedge portfolio at time zero is:

$$
P P_{0}=\sum_{t=1}^{T} E_{D}(t) \cdot B S P_{0}(t)+E_{S}(T) \cdot B S P_{0}(T)
$$

where $E_{D}(t)$ is the expected number of deaths at the end of the month $t$ and $E_{S}(t)$ is the expected number of survivors at the maturity date of the contract $T$.

We assume that $\left\{N_{D}(t)\right\}_{t=1}^{T} \cup\left\{N_{S}(T)\right\}$ is multinomial with parameters $\left(C ; q_{x}, \cdots,{ }_{T-1 \mid} q_{x},{ }_{n} p_{x}\right)$ where $C$ is the number of policies issued at time zero, ${ }_{t \mid} q_{x}$ is the probability that a life aged $x$ at issue dies in $[t-1, t]$ and ${ }_{T} p_{x}$ is the probability that a life aged $\mathrm{x}$ at issue is alive at time $T$.

So we have that $E_{D}(t)=C \cdot{ }_{t-1 \mid} q_{x}$ and $E_{S}(t)=C \cdot{ }_{T} p_{x}$

As aforementioned, we calculate the quantity $B S P_{0}$ in (27) by an adaptation of the standard Black-Scholes formula.

We need to modify the standard formula for the put options to reflect the fact that the underlying asset is not the stock price itself, but the fund value, and this differs from the stock price through the deduction of the management charges. 
Net of the monthly management charges the fund is:

$$
F_{t}=F_{0} \cdot \frac{S_{t}}{S_{0}} \cdot(1-m)^{t}
$$

where $S_{t}$ is the stock index.

Let $F_{0}=S_{0}$, then the option price with maturity $t$ is:

$$
B S P_{0}(t)=e^{-r \cdot t} \cdot E_{Q}\left[\left(G_{t}-S_{t} \cdot(1-m)^{t}\right)_{+}\right]
$$

Using this approach, we replace $S_{0}$ by $S_{0} \cdot(1-m)^{t}$ in the standard Black-Scholes formula. Then the put option price is computed as follows:

$$
B S P_{0}(t)=G_{t} \cdot e^{-r \cdot t} \cdot \Phi\left(-d_{2}\right)-S_{0} \cdot(1-m)^{t} \cdot \Phi\left(-d_{1}\right)
$$

where $r$ is the risk-free (force) of interest, $\Phi(\bullet)$ denotes the cumulative normal density function, $G_{t}=P^{\prime} \cdot e^{g . t}$ is the increasing strike price, $m$ is the monthly charge and

$$
\begin{gathered}
d_{1}=\frac{\log \left(S_{0} \cdot(1-m)^{t} / G_{t}\right)+\left(r+\sigma_{S}^{2} / 2\right) \cdot t}{\sigma \cdot \sqrt{t}}, \\
d_{2}=d_{1}-\sigma \cdot \sqrt{t},
\end{gathered}
$$

$\sigma_{s}^{2}$ is the estimated volatility of the asset return.

If we model the financial variables by a two-dimensional VAR model, $\sigma_{S}^{2}$ is the estimated volatility of the weighted continuously compounded rate of return $\delta_{t}$. It assumes different values whether the basket of assets is rebalanced or not (Section 3.1)

Modelling the assets by a $(n+1)$-dimensional VAR (1) model, $\sigma_{S}^{2}$ is the weighted volatility of the $n$ returns on assets, that is $\sigma_{S}^{2}=\sum_{i=1}^{n} \sum_{j=1}^{n} \omega_{i} \omega_{j} \sigma_{i, j}$.

Let us now consider the case in which the initial allocation does not change and the financial variables are modelled by a $(n+1)$-dimensional VAR (1) model.

In this case, at time zero, the insurer buys a portfolio of basket options.

As it is well known, a basket option is an option on a basket of assets, typically stocks.

In this case, the Fund value in (28) can be modified as follows:

$$
F_{t}=\sum_{i=1}^{n} \omega_{i} \cdot F_{0}^{i} \cdot \frac{S_{t}^{i}}{S_{0}^{i}} \cdot(1-m)^{t}
$$

where $S_{t}^{i}$ is the $i$-th stock index, $\omega_{i}$ is the proportion of the fund invested in asset $i$.

Letting $C \cdot F_{0}^{i}=S_{0}^{i}$, we can write:

$$
F_{t}=\sum_{i=1}^{n} \omega_{i} \cdot S_{t}^{i} \cdot(1-m)^{t} .
$$

Our interest is in the dynamics of the weighted arithmetic sum of the underlying $S_{t}^{i}(i=1,2,3, \cdots, n)$. Similar to a plain vanilla option, the value of a basket option is equal to its expected payoff discounted at the risk free rate. The 
expectation is computed with respect to the state-price density function (SPD) also known as the risk neutral probability density function. The SPD, in this case, is not known and closed form solutions to the problem are not available. A common approach is to estimate the state density function by a lognormal functional form, using different matching techniques. One way is to compute the first two moments of the basket's payoff structure at maturity and then match them to those of the lognormal distribution.

Following [9], we define the "pseudo-forward" time t of the basket:

$$
A_{t}=\sum_{i=1}^{n} \omega_{i} A_{t}^{i}
$$

where $A_{t}^{i}=S_{0}^{i} \cdot(1-m)^{t} \cdot e^{r \cdot t}$, being $r$ the risk free rate.

Dividing $F_{t}$ by $A_{t}$ and denoting the ratio by $F_{t}^{*}$, we normalize it to have a mean of 1 (i.e. $M_{1}=1$ ).

The second moment of $F_{t}^{*}$ is:

$$
M_{2}=\frac{1}{A_{t}^{2}} \cdot \sum_{i=1}^{n} \sum_{j=1}^{n} \omega_{i} \omega_{j} A_{i} A_{j} e^{\rho_{i, j} \sigma_{i} \sigma_{j} t}
$$

where $\rho_{i, j}$ is the correlation coefficient between $A_{i}$ and $A_{j}$.

Assuming that the normalized basket $F_{t}^{*}$ is lognormal with mean $M_{1}=1$, it follows that the variance of the basket is

$$
v=\ln \left(M_{2}\right)
$$

The moment-matched lognormal state-price density function gives the price of the put option, by means of the usual Black-Scholes formula:

$$
\operatorname{LNBSP}_{0}(t)=G_{t} \cdot e^{-r \cdot t} \cdot \Phi\left(-d_{2}\right)-A_{t} \cdot \Phi\left(-d_{1}\right)
$$

where $\Phi(\bullet)$ is the cumulative normal density function and

$$
\begin{gathered}
d_{1}=\frac{\log \left(A_{t} \cdot(1-m)^{t} / G_{t}\right)+v / 2}{\sqrt{v}} \\
d_{2}=d_{1}-\text { sqrtv }
\end{gathered}
$$

Equation (27) becomes

$$
P P_{0}=\sum_{t=1}^{T} E_{D}(t) \cdot L N B S P_{0}(t)+E_{S}(T) \cdot L N B S P_{0}(T)
$$

\subsection{The Portfolio Value at Inception}

The portfolio value at time zero is given by the difference between the random inflows and outflows arising from the contract.

Let us now consider the inflows connected to the contract.

They are given by the expense charge paid at issue by each insured as a percentage $c$ of the premium $P$ plus the present value of the collected monthly charges $m$ to the insured's account. Denoting by $I F_{0}$ the inflows evaluated at time zero, in the case of a two-dimensional VAR (1) model and referring to (19) 
and (20) we have:

$$
\begin{aligned}
I F_{0}= & C \cdot P \cdot c+\sum_{t=1}^{T}\left(N_{D}(t) \cdot\left(1-(1-m)^{t}\right) \cdot P^{\prime} \cdot e^{\sum_{s=1}^{t} \delta_{s}} \cdot e^{-\sum_{s=1}^{t} r_{s}}\right) \\
& +N_{S}(T) \cdot\left(1-(1-m)^{\mathrm{T}}\right) \cdot P^{\prime} \cdot e^{\sum_{s=1}^{t} \delta_{s}} \cdot e^{-\sum_{s=1}^{t} r_{s}}
\end{aligned}
$$

where the $\delta_{s}$ values depend on rebalancing or not and on the investment strategy (see Section 3.1).

In the case of the $(n+1)$ dimensional VAR (1) model, using Equations (24)-(26), we get:

1) Rebalancing case:

$$
\begin{aligned}
I F_{0}= & C \cdot P \cdot c+\sum_{t=1}^{T}\left(N_{D}(t) \cdot\left(1-(1-m)^{t}\right) \cdot P^{\prime} \cdot e^{\sum_{s=1}^{t} \sum_{i=1}^{n} \omega_{i} \cdot \delta_{s}^{i}} \cdot e^{-\sum_{s=1}^{t} r_{s}}\right) \\
& +N_{S}(T) \cdot\left(1-(1-m)^{\mathrm{T}}\right) \cdot P^{\prime} \cdot e^{\sum_{s=1}^{T} \sum_{i=1}^{n} \omega_{i} \cdot \delta_{s}^{i}} \cdot e^{-\sum_{s=1}^{T} r_{s}}
\end{aligned}
$$

2) No Rebalancing case:

$$
\begin{aligned}
I F_{0}= & C \cdot P \cdot c+\sum_{t=1}^{T} \sum_{i=1}^{n}\left(N_{D}(t) \cdot\left(1-(1-m)^{t}\right) \cdot \omega_{i} \cdot P^{\prime} \cdot e^{\sum_{s=1}^{t} \delta_{s}^{i}} \cdot e^{-\sum_{s=1}^{t} r_{s}}\right) \\
& +N_{S}(T) \cdot\left(1-(1-m)^{\mathrm{T}}\right) \cdot \omega_{i} \cdot P^{\prime} \cdot e^{\sum_{s=1}^{T} \delta_{s}^{i}} \cdot e^{-\sum_{s=1}^{T} r_{s}} .
\end{aligned}
$$

In (43) and (44) $\omega_{i}, i=1,2, \cdots, n$, are the proportions invested in each of the $n$ assets and their value changes according to the chosen investment strategy.

Let us now consider the cash flows for each $t$, connected to the contract. They are given by the difference between the obligations arising from the guarantees (linked to the actual number of deaths each month (GMDB) and to the actual number of survivors at time $T(\mathrm{GMMB})$ ) and the inflows arising from the puts payoff. At time zero we get:

$$
\begin{aligned}
& C F_{0}(G M D B)=\sum_{t=1}^{T}\left(N_{D}(t)-E_{D}(t)\right) \cdot\left(G_{t}-F_{t}, 0\right)_{+} \cdot e^{-\sum_{s=1}^{t} r_{s}} \\
& C F_{0}(G M M B)=\left(N_{S}(t)-E_{S}(T)\right) \cdot\left(G_{T}-F_{T}, 0\right)_{+} \cdot e^{-\sum_{s=1}^{T} r_{s}} .
\end{aligned}
$$

Therefore, the portfolio value at inception is:

$$
V P_{0}=I F_{0}-P P_{0}-C F_{0}(G M D B)-C F_{0}(G M M B)
$$

where the value of each quantity depends on the specific model and investment strategy, as previously explained.

We are interested in capturing the distribution of the potential future markets and mortality rates. To this aim we resort to a Monte Carlo simulation, assessing $V P_{0}$ across a real world scenario.

\section{Illustrations}

In this section we study an illustrative portfolio of VA contracts offering GMDB and GMMB guarantees (see Section 2) issued to 1000 independent lives aged 55 and paying a single premium $\mathrm{P}=6000$ at issue. 
Table 1 summarizes the portfolio characteristics.

The premium $P$, net of the initial charge ( $3 \%$ of $P$ ), is invested into the fund. The benefits are paid monthly and a monthly charge $m=0.0025$ is deducted. The guaranteed amount is obtained by accumulating the net premium at the guaranteed rate g. In our example we consider three different guarantee rates: $0.015,0.02$ and 0.025 . We assume that the VA writer offers two asset allocations to the policyholders. The first one, called moderate, is characterized by a large proportion of assets invested in bonds. The second one, mixed, is characterized by equal proportions in bonds and stocks. These asset allocations characterize some funds available in VA products sold in Europe (among others the "Accumulator" sold by AXA)

According to the moderate allocation (say Allocation A) we assume that the Company invests $70 \%$ in the Euro Big index, $18 \%$ in the Eurostoxx index and $12 \%$ in the $\mathrm{S} \& \mathrm{P}$ index. For the mixed allocation (say Allocation $\mathrm{B}$ ) the fund allocation is: $50 \%$ in the Euro Big index, the $30 \%$ in the Eurostoxx index and the $20 \%$ in the $\mathrm{S} \& \mathrm{P}$ index.

Moreover we use two different accumulation functions to calculate the expected returns on investment in each fund, depending on whether the basket of assets constituting the fund itself is rebalanced or not.

The Euro Big index (Broad investment Euro BIG Bond index) provides a well known benchmark for Euro Bonds fixed-income portfolios. It covers all sectors of the investment grade fixed income market that are accessible to institutional investors and accurately measures their performance and characteristics. The Eurostoxx index is a broad, yet liquid, subset of the Stoxx Europe 600 Index. With a variable number of components, the index represents large, mid and small capitalization companies of 12 Eurozone countries: Austria, Belgium, Finland, France, Germany, Greece, Ireland, Italy, Luxembourg, the Netherlands, Portugal and Spain. The S \& P500 is a free-capitalization-weighted index of the

Table 1. The illustrative portfolio.

\begin{tabular}{ccc}
\hline Premium & $\boldsymbol{P}$ & 600 \\
Monthly charge & $\boldsymbol{m}$ & $\mathbf{0 . 0 0 2 5}$ \\
Initial charge & $\boldsymbol{c}$ & $3 \%$ \\
\hline Guaranteed rates & $\mathrm{g}$ & $1.5 \%$ \\
\hline Moderate allocation & Allocation A & $2 \%$ \\
\hline Mixed allocation & & $18 \%$ Eurostoxx, \\
\hline
\end{tabular}


prices of 500 large-cap common stocks actively traded in the United States.

The discounting rate is modelled using the Italian "Rendistato" that is a weighted average yield on a basket of Italian government securities.

For each index, we use the data from June 2002 to April 2016. The data sources are: the yield book of Citi fixed income indices for the EuroBIG index, Yahoo finance for the Eurostoxx and the S \& P500 indices and the historical series published by the Bank of Italy, for the Italian Rendistato.

Figure 1 illustrates the behavior of the three considered indexes as well as the Italian Rendistato series.

For the mortality rates, we refer to a non parametric mortality table: IPS55

\subsection{Estimation of the VAR Model Parameters}

The parameter estimation results are obtained using the VAR package of the software R. All the parameters are estimated on a monthly basis.

Before estimating the parameters, we subtract the long term mean vector $\underline{\mu}$ from the data. We consider the sample mean of each series as an estimator for the long term mean vector $\underline{\mu}$.

Let us first consider the case of the two-dimensional VAR (1) model described in Section 3.1. The long term mean of the discounting rate is 0.0028 . Table 2 shows the long term mean of the composite rate of return on assets in each case: "rebalancing" for allocation A and B (column 1) and "not rebalancing" for allocation A and B (column 2).

Table 3 and Table 4 show the parameter estimation results for Allocation A. We have a process that is stable since the eigenvalues of the $\underline{\Phi}$ matrix are less than one. They are $(0.9929,0.0977)$ for the rebalancing case and $(0.9929,0.0957)$
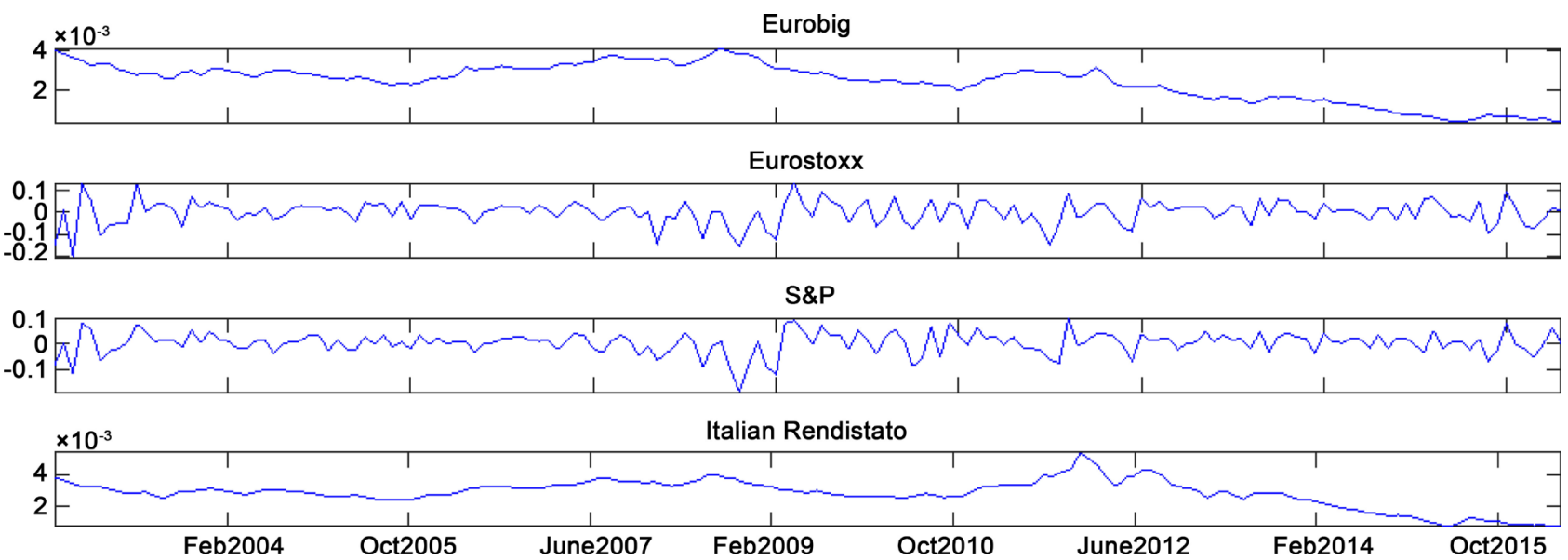

Figure 1. EuroBIG, Eurostoxx, S \& P 500 and Italian Rendistato series. June 2002-April 2016.

Table 2. $\mu$ estimation. Two-dimensional VAR (1).

\begin{tabular}{cccc}
\hline \multicolumn{2}{c}{ Rebalancing } & \multicolumn{2}{c}{ Not Rebalancing } \\
\hline Allocation A & Allocation B & Allocation A & Allocation B \\
\hline 0.0019 & 0.0014 & 0.0021 & 0.0015 \\
\hline
\end{tabular}


in case of no rebalancing.

Table 5 and Table 6 show the parameter estimation results for Allocation B. We have a process that is stationary since the eigenvalues of the $\Phi$ matrix are less than one. In this case they are $(0.9929,0.1009)$ for the rebalancing case and $(0.9929,0.1139)$ in case of no rebalancing.

Let us now consider the $(n+1)$-dimensional VAR ( 1$)$ model: in the specific example we are considering $n=3$, therefore we will have a four-dimensional VAR (1) model.

We indicate by $\delta_{t}^{1}$ the Eurobig index, by $\delta_{t}^{2}$ the Eurostoxx50 index, by $\delta_{t}^{3}$ the S \& P index and by $r_{t}$ the Italian Rendistato (Figure 1 ).

Table 3. $\Phi$ matrix estimation. Allocation A (moderate). Two-dimensional VAR (1) model for the continuous weighted average rates of return in rebalancing and not rebalancing case and Italian Rendistato series. June 2002-April 2016.

\begin{tabular}{ccccc}
\hline & \multicolumn{2}{c}{ Rebalancing } & \multicolumn{2}{c}{ Not Rebalancing } \\
\hline & $\delta_{t}$ & $r_{t}$ & $\delta_{t}$ & $r_{t}$ \\
\hline$\delta_{t}$ & 0.9720 & 0.56194 & 0.09051 & 0.72280 \\
$r_{t}$ & -0.00079 & 0.99340 & -0.00080 & 0.99355 \\
\hline
\end{tabular}

Table 4. $\underline{\Sigma}_{a}$ matrix estimation. Allocation A (moderate). Two-dimensional VAR (1) model for the continuous weighted average rates of return in rebalancing and not rebalancing case and Italian Rendistato series. June 2002-April 2016.

\begin{tabular}{ccccc}
\hline & \multicolumn{2}{c}{ Rebalancing } & \multicolumn{2}{c}{ Not Rebalancing } \\
\hline & $\delta_{t}$ & $r_{t}$ & $\delta_{t}$ & $r_{t}$ \\
\hline$\delta_{t}$ & $2.110 \mathrm{e}-04$ & $4.165 \mathrm{e}-08$ & $1.718 \mathrm{e}-04$ & $-1.693 \mathrm{e}-08$ \\
$r_{t}$ & $4.165 \mathrm{e}-08$ & $4.018 \mathrm{e}-08$ & $-1.693 \mathrm{e}-08$ & $4.020 \mathrm{e}-08$ \\
\hline
\end{tabular}

Table 5. $\Phi$ matrix estimation. Allocation B (mixed). Two-dimensional VAR (1) model for the continuous weighted average rates of return in rebalancing and not rebalancing case and Italian Rendistato series. June 2002-April 2016.

\begin{tabular}{ccccc}
\hline & \multicolumn{2}{c}{ Rebalancing } & \multicolumn{2}{c}{ Not Rebalancing } \\
\hline & $\delta_{t}$ & $r_{t}$ & $\delta_{t}$ & $r_{t}$ \\
\hline$\delta_{t}$ & 0.10068 & 0.42730 & 0.10056 & 0.55718 \\
$r_{t}$ & -0.00048 & 0.99313 & -0.00049 & 0.99321 \\
\hline
\end{tabular}

Table 6. $\underline{\Sigma}_{a}$ matrix estimation. Allocation B (mixed). Two-dimensional VAR (1) model for the continuous weighted average rates of return in rebalancing and not rebalancing case and Italian Rendistato series. June 2002-April 2016.

\begin{tabular}{ccccc}
\hline & \multicolumn{2}{c}{ Rebalancing } & \multicolumn{2}{c}{ Not Rebalancing } \\
\hline$\delta_{t}$ & $r_{t}$ & $\delta_{t}$ & $r_{t}$ \\
\hline$\delta_{t}$ & $5.876 \mathrm{e}-04$ & $5.392 \mathrm{e}-08$ & $4.819 \mathrm{e}-04$ & $8.495-09$ \\
$r_{t}$ & $5.392 \mathrm{e}-08$ & $4.017 \mathrm{e}-08$ & $8.495 \mathrm{e}-09$ & $4.019 \mathrm{e}-08$ \\
\hline
\end{tabular}


The long term means on the three assets are: $\mu^{1}=0.0024, \mu^{2}=0.0022$, $\mu^{3}=0.0044$. The estimation results for the $\Phi$ matrix and the covariance matrix of the residuals are shown in Table 7 and Table 8.

The process is stationary since the absolute values of the eigenvalues of the $\Phi$ matrix, which are $0.9919,0.9513,0.1005$ and 0.09064 , are less than one.

Nevertheless let us look at Table 7. Each row shows the regression parameters of the equations describing the evolution in time of each variable.

Looking at the first and second rows, first of all we observe a strong autoregressive dependence of the two bond indexes, $\delta_{t}^{2}$ and $r_{t}$. Indeed the parameters explaining the evolution based on their own lag are high: 0.98287 and 0.95810 , respectively. The regression parameters explaining the dependence on the other variables show a weak dependence on stock indexes.

The third and fourth rows of Table 7 give the estimated parameters of stock indexes processes. The regression parameters explaining the dependence on the lag of bond indexes are: -10.70956 and 9.01693 for $\delta_{t}^{2}$ equation and -9.95162 and 9.77489 for the $r_{t}$ equation.

All these observations tell us that there is not a clear dependence of bond indexes $\left(\delta^{2}\right.$ and $r_{t}$ ) on stock indexes $\left(\delta^{2}\right.$ and $\left.\delta^{3}\right)$.

Let us now focus on stock indexes. The two indexes show a weak autoregressive dependence: -0.038769 and 0.05082 , respectively. In order to better capture their mutual dependence, we modelled the two indexes by a two-dimensional VAR (1) model. The estimated parameters are shown in Table 9.

The autoregressive dependence of each index is still weak. Moreover the eigenvalues are low: 0.1322 and 0.06405 .

Based on the aforementioned observations, we conclude that a VAR (1) is questionable when interested in describing the behavior of stock indexes.

We decide to model the stock indexes by a Geometric Brownian Motion model (GBM), the most widely used model for stock prices.

As it is well known, the stock indexes $S_{t}$ follows a GBM if:

$$
\mathrm{d} S_{t}=\mu S_{t} \mathrm{~d} t+\sigma S_{t} \mathrm{~d} W_{t}
$$

where $W_{t}$ is a Brownian motion and $\mu$ and $\sigma$ are positive constants.

This implies that the instantaneous rate of return $\delta_{t}$ is a Brownian Motion:

$$
\delta_{t}=\frac{\mathrm{d} S_{t}}{S_{t}}=\mu+\sigma \mathrm{d} W_{t} .
$$

Table 7. $\Phi$ matrix estimation. Four-dimensional VAR (1) model. Eurobig, Eurostoxx 50, SP and Italian Rendistato series. June 2002-April 2016.

\begin{tabular}{ccccc}
\hline & $\delta_{t}^{1}$ & $r_{t}$ & $\delta_{t}^{2}$ & $\delta_{t}^{3}$ \\
\hline$\delta_{t}^{1}$ & 0.0 .98287 & 0.00739 & -0.00019 & 0.00018 \\
$r_{t}$ & 0.03965 & 0.95810 & -0.00068 & 0.00067 \\
$\delta_{t}^{2}$ & -10.14948 & 8.45684 & -0.03876 & 0.13267 \\
$\delta_{t}^{3}$ & -9.95162 & 9.77489 & 0.05441 & 0.05082 \\
\hline
\end{tabular}


Table 8. $\underline{\Sigma}_{a}$ matrix estimation. Four-dimensional VAR (1) model. Eurobig, Eurostoxx50, SP and Italian Rendistato series. June 2002-April 2016.

\begin{tabular}{ccccc}
\hline & $\delta_{t}^{1}$ & $r_{t}$ & $\delta_{t}^{2}$ & $\delta_{t}^{3}$ \\
\hline$\delta_{t}^{1}$ & $2.188 \mathrm{e}-08$ & $1.389 \mathrm{e}-08$ & $1.024 \mathrm{e}-07$ & $-1.732 \mathrm{e}-09$ \\
$r_{t}$ & $1.389 \mathrm{e}-08$ & $3.990 \mathrm{e}-08$ & $1.689 \mathrm{e}-07$ & $2.291 \mathrm{e}-07$ \\
$\delta_{t}^{2}$ & $9.292 \mathrm{e}-08$ & $1.459 \mathrm{e}-07$ & $2.054 \mathrm{e}-03$ & $1.851 \mathrm{e}-03$ \\
$\delta_{t}^{3}$ & $-1.732 \mathrm{e}-09$ & $2.291 \mathrm{e}-07$ & $1.918 \mathrm{e}-03$ & $1.753 \mathrm{e}-03$ \\
\hline
\end{tabular}

Table 9. $\Phi$ matrix estimation. Two-dimensional VAR (1) model. Eurostoxx 50 and S \& P series. June 2002-April 2016.

\begin{tabular}{ccc}
\hline & $\delta_{t}^{2}$ & $\delta_{t}^{3}$ \\
\hline$\delta_{t}^{2}$ & -0.03738 & 0.15135 \\
$\delta_{t}^{3}$ & 0.029879 & 0.10566 \\
\hline
\end{tabular}

The solution we propose in order to include the GBM in the model, is a modified version of the four-dimensional VAR (1) model.

First of all, we estimate again the parameters of a two-dimensional VAR (1) model considering only the bond indexes, Eurobig and Rendistato.

The $\Phi$ matrix estimation results are given in Table 10 .

Again the process is stationary because the eigenvalues of the $\Phi$ matrix are 0.992 and 0.9512 .

Let us now come back to the four-dimensional VAR (1) model. We replace the estimated $\Phi$ matrix in Table 7, with the $\underline{\Phi}^{*}$ matrix of Table 11.

The $\Phi^{*}$ matrix is obtained by setting all the parameters connected to the Eurostoxx and the S \& P equal to zero and by setting the other parameters equal to the ones given in Table 10 .

With reference to (11) the residuals matrix is obtained as follows:

$$
\underline{a}^{*}=\left(\underline{X_{t}}-\underline{\mu}\right)-\underline{\Phi}^{*} \cdot\left(\underline{X_{t-1}}-\underline{\mu}\right)
$$

where $\underline{X}_{t}$ is given by (21).

It is than possible to estimate the covariance matrix of the residuals $\underline{\Sigma}_{a}^{*}$ (see Table 11).

In order to compare the VAR (1) model and the proposed modified version, we ran 10,000 simulations for values of $\delta_{t}^{1}, \delta_{t}^{2}, \delta_{t}^{3}$ and $r_{t}$ values with $t \in[1,2, \cdots, 120]$ assuming that their evolution in time is described by the 'full' four-dimensional VAR (1) model (Table 7 and Table 8) and by the modified version of the same model (Table 11 and Table 12).

Figure 2 and Figure 3 show the evolution over time of the expected values of the four variables in both cases and for different selected initial values. For each variable and for each $t$, the expected values are computed as the mean of the 10,000 simulated values.

We observe that the two processes have the same long term dynamics with different behavior only for a few months. 
Table 10. $\Phi$ matrix estimation. Two-dimensional VAR (1) model. Eurobig and Italian Rendistato series. June 2002-April 2016.

\begin{tabular}{ccc}
\hline & $\delta_{t}^{1}$ & $r_{t}$ \\
\hline$\delta_{t}^{1}$ & 0.98443 & 0.01244 \\
$r_{t}$ & 0.04013 & 0.95873 \\
\hline
\end{tabular}

Table 11. $\Phi$ matrix estimation. Four-dimensional VAR (1) model. Eurobig and Italian Rendistato series. June 2002-April 2016.

\begin{tabular}{ccccc}
\hline & $\delta_{t}^{1}$ & $r_{t}$ & $\delta_{t}^{2}$ & $\delta_{t}^{3}$ \\
\hline$\delta_{t}^{1}$ & 0.98443 & 0.01244 & 0 & 0 \\
$r_{t}$ & 0.04013 & 0.95873 & 0 & 0 \\
$\delta_{t}^{2}$ & 0 & 0 & 0 & 0 \\
$\delta_{t}^{3}$ & 0 & 0 & 0 & 0 \\
\hline
\end{tabular}

Table 12. $\underline{\Sigma}_{a}^{*}$ matrix estimation. Modified four-dimensional VAR (1) model. Eurobig, Eurostoxx50, SP and Italian Rendistato series. June 2002-April 2016.

\begin{tabular}{ccccc}
\hline & $\delta_{t}^{1}$ & $r_{t}$ & $\delta_{t}^{2}$ & $\delta_{t}^{3}$ \\
\cline { 2 - 5 }$\delta_{t}^{1}$ & $2.1370 \mathrm{e}-08$ & $1.3620 \mathrm{e}-08$ & $7.8776 \mathrm{e}-08$ & $-4.1885 \mathrm{e}-08$ \\
$r_{t}$ & $1.3620 \mathrm{e}-08$ & $3.9234 \mathrm{e}-08$ & $1.6267 \mathrm{e}-08$ & $3.9234 \mathrm{e}-08$ \\
$\delta_{t}^{2}$ & $7.8776 \mathrm{e}-08$ & $1.6267 \mathrm{e}-08$ & 0.0029 & 0.0019 \\
$\delta_{t}^{3}$ & $-4.1887 \mathrm{e}-08$ & $1.5296 \mathrm{e}-07$ & 0.0019 & 0.0018 \\
\hline
\end{tabular}
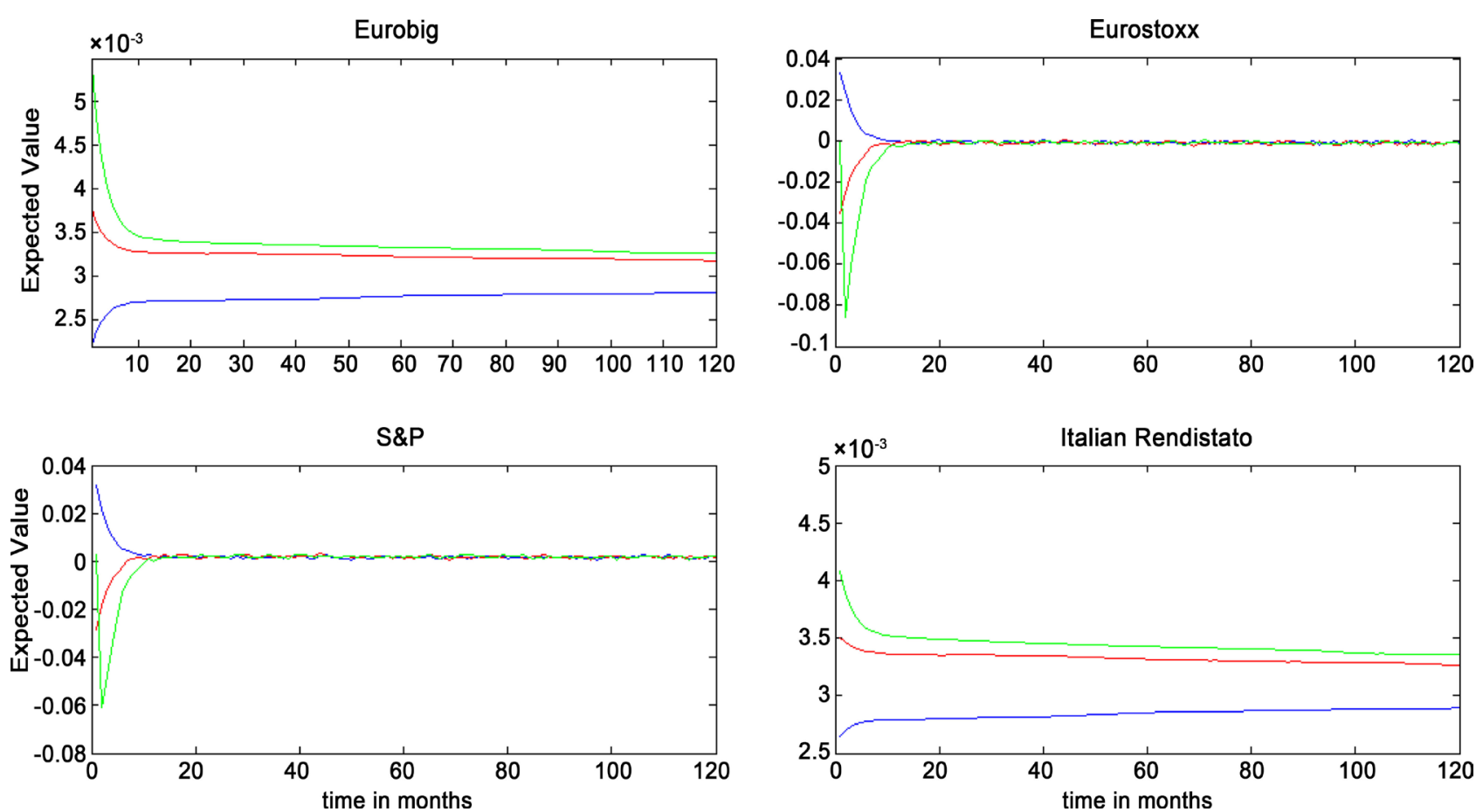

Figure 2. Four-dimensional VAR (1) model. Expected values with different initial values. Number of simulations $=10,000$. 

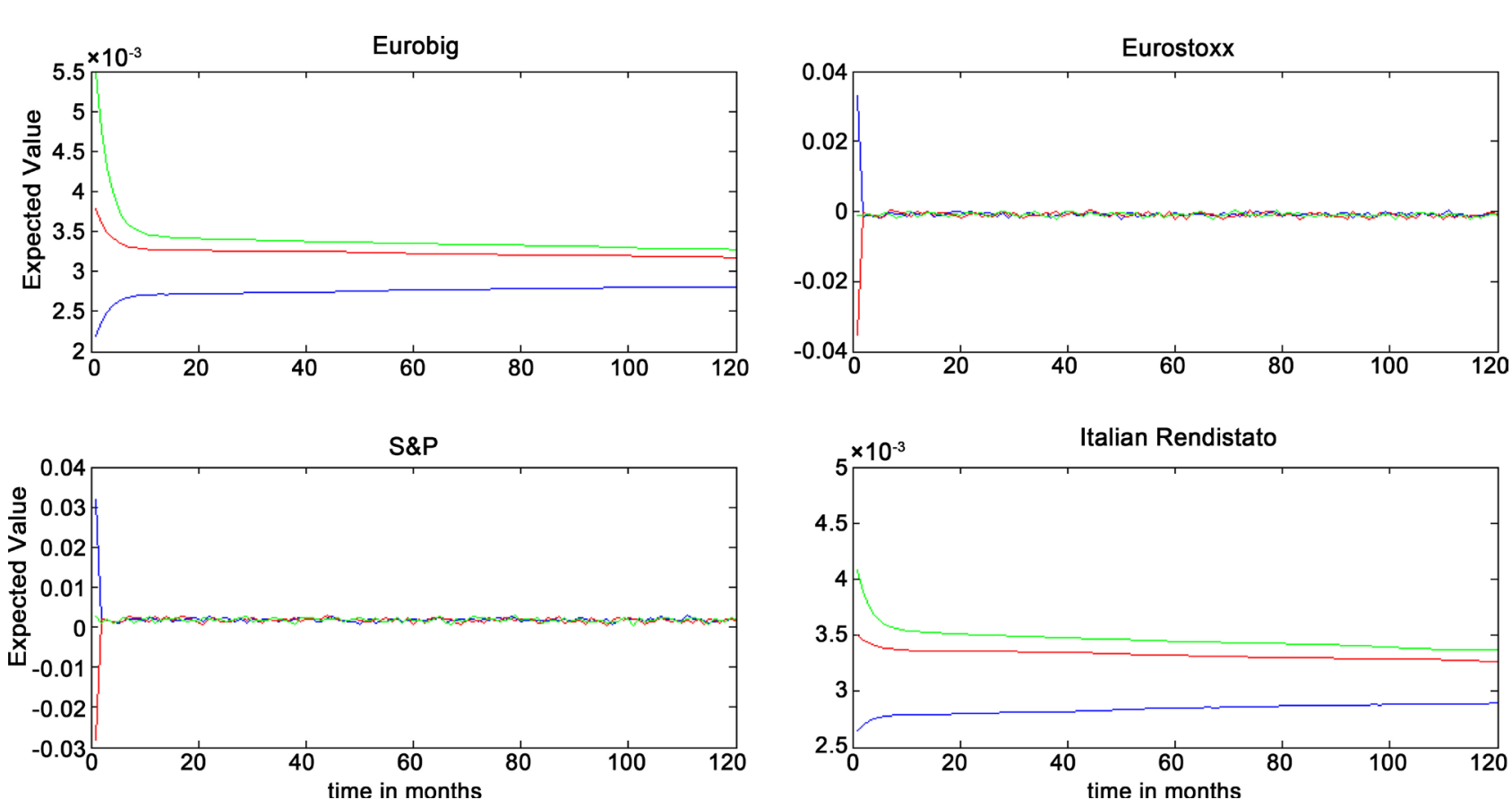

Figure 3. Modified four-dimensional VAR (1) model. Expected values with different initial values. Number of simulations = 10,000 .

\subsection{Some Results}

In this section, we refer to the illustrative portfolio described in Section 5.1.

We are interested in the expected value and the conditional value at risk at a chosen confidence level of $95 \%$ of the portfolio at inception, for a static hedging strategy (Section 4.2). The software used for the following analysis is Matlab.

We resort to a Monte Carlo simulation, assessing the quantities of interest across a real-world scenario set for financial and mortality variables.

Here are the steps of the simulation procedure:

1) Randomly generate the monthly rates of return and the monthly discounting rates using the results of the estimated parameters;

2) Randomly generate the monthly death probabilities. For fractional ages a Uniform distribution of deaths assumption is made: for example the probability of dying in any month of a given year is the same.

3) Assess $V P_{0}$ value (Equation (47));

4) Repeat the procedure $N=10,000$ times;

5) Estimate $E\left[V P_{0}\right]$ by taking the sample mean of the $\mathrm{N}$ simulated values of $V P_{0}$;

6) Estimate the conditional value at risk at a 95\% confidence level from the empirical distribution of $V P_{0}$. As is well known, the $C V a R(1-\alpha) \%$ is the average of the $(1-\alpha) \%$ worst scenarios. In our example we choose $\alpha=95 \%$ and the CVaR $95 \%$ is the average of the $5 \%$ lowest values of $V P_{0}$.

The simulation procedure is repeated for each assumption about the financial variables previously described, the two and four-dimensional VAR (1) models, both with rebalancing of the basket of assets and without rebalancing. 
We then compare the results with the choice of no hedge, that is the case in which the insurer decides not to buy the hedge portfolio of put options.

Table 13 shows the cost of the hedging portfolio in each case. The portfolio price has been determined in a risk neutral framework on the basis of the results obtained in Sections 4.1 and 5.1.

First of all we observe that in each case the cost is lower than the intitial charge paid by the insurers, that is $C * P * c$, that in our illustrative portfolio amounts to 180,000 euros.

As the guaranteed rate gets higher, the put options price increase and the cost of the entire portfolio increases, too.

In general, the cost of the hedging portfolio is higher for the rebalancing strategy than the no rebalancing one, as expected. Indeed, the estimated volatilities of the underlying are higher in the rebalancing case, for each considered option.

The same happens going from Allocation A to Allocation B. The second strategy is more risky because it is characterized by a higher proportion of assets invested in stocks and therefore a higher volatility.

Tables 14-17 compare the results for static hedging and those for when the Company decides not to hedge at all.

First of all, we observe that in each considered case the expected value of the portfolio $E\left[V P_{0}\right]$ is higher if we shift from no hedging to static hedging. This is expected because the no hedging case does not "adapt" to mortality experience over time, as static hedging does to some extent.

Moreover we observe that Allocation B gives lower values of $E\left[V P_{0}\right]$ and that Table 13. Cost of the hedging Portfolio.

\begin{tabular}{|c|c|c|c|}
\hline \multirow{4}{*}{ 2D VAR (1) Rebalancing } & $g$ & Allocation $A$ & Allocation $B$ \\
\hline & $1.4 \%$ & 23,950 & 42,000 \\
\hline & $1.5 \%$ & 27,430 & 54,970 \\
\hline & $2 \%$ & 52,140 & 110,520 \\
\hline \multirow{4}{*}{ 2D VAR (1) No Rebalancing } & $g$ & Allocation $A$ & Allocation $B$ \\
\hline & $1.4 \%$ & 18,330 & 36,800 \\
\hline & $1.5 \%$ & 21,200 & 45,480 \\
\hline & $2 \%$ & 41,820 & 90,780 \\
\hline \multirow{4}{*}{ 4D VAR (1) Rebalancing } & $g$ & Allocation $A$ & Allocation $B$ \\
\hline & $1.4 \%$ & 22,990 & 41,180 \\
\hline & $1.5 \%$ & 26,390 & 52,900 \\
\hline & $2 \%$ & 50,560 & 107,240 \\
\hline \multirow{4}{*}{ 4D VAR (1)No Rebalancing } & $g$ & Allocation $A$ & Allocation $B$ \\
\hline & $1.4 \%$ & 17,500 & 34,120 \\
\hline & $1.5 \%$ & 19,880 & 43,480 \\
\hline & $2 \%$ & 39,010 & 87,030 \\
\hline
\end{tabular}


Table 14. Simulation results for $V P_{0}: 2 \operatorname{DVAR}(1)$, Rebalancing case, $N=10,000$, $\alpha=5 \%$.

\begin{tabular}{|c|c|c|c|}
\hline \multirow{6}{*}{$g=1.4 \%$} & NO HEDGE & $E\left[V P_{0}\right]$ & $C V a R_{V P}(\alpha)$ \\
\hline & Allocation $A$ & 36,280 & $-990,400$ \\
\hline & Allocation $B$ & $-226,780$ & $-1,833,000$ \\
\hline & STATIC HEDGING & & \\
\hline & Allocation A & 261,570 & 182,190 \\
\hline & Allocation $B$ & 255,250 & 124,550 \\
\hline \multirow{6}{*}{$g=1.5 \%$} & NO HEDGE & & \\
\hline & Allocation $A$ & 29,500 & $-1,071,900$ \\
\hline & Allocation $B$ & $-250,620$ & $-1,867,000$ \\
\hline & STATIC HEDGING & & \\
\hline & Allocation $A$ & 257,920 & 177,380 \\
\hline & Allocation B & 247,120 & 116,860 \\
\hline \multirow{6}{*}{$g=2 \%$} & NO HEDGE & & \\
\hline & Allocation $A$ & $-119,650$ & $-1,337,400$ \\
\hline & Allocation $B$ & $-389,120$ & $-2,127,200$ \\
\hline & STATIC HEDGING & & \\
\hline & Allocation $A$ & 233,780 & 153,600 \\
\hline & Allocation $B$ & 193,760 & 62,550 \\
\hline
\end{tabular}

Table 15. Simulation results for $V P_{0}: 2 \operatorname{DVAR}(1)$, No Rebalancing case, $N=10,000$, $\alpha=5 \%$.

\begin{tabular}{|c|c|c|c|}
\hline \multirow{6}{*}{$g=1.4 \%$} & NO HEDGE & $E\left[V P_{0}\right]$ & $C V a R_{V P}(\alpha)$ \\
\hline & Allocation $A$ & 104,360 & $-817,600$ \\
\hline & Allocation $B$ & $-173,380$ & $-1,653,300$ \\
\hline & STATIC HEDGING & & \\
\hline & Allocation $A$ & 273,960 & 200,830 \\
\hline & Allocation $B$ & 267,320 & 146,940 \\
\hline \multirow{6}{*}{$g=1.5 \%$} & NO HEDGE & & \\
\hline & Allocation $A$ & 86,100 & $-856,600$ \\
\hline & Allocation $B$ & $-192,580$ & $-1,689,800$ \\
\hline & STATIC HEDGING & & \\
\hline & Allocation $A$ & 271,590 & 198,990 \\
\hline & Allocation $B$ & 256,390 & 137,020 \\
\hline \multirow{6}{*}{$g=2 \%$} & NO HEDGE & & \\
\hline & Allocation $A$ & $-31,720$ & $-1,176,000$ \\
\hline & Allocation $B$ & $-323,490$ & $-1,943,800$ \\
\hline & STATIC HEDGING & & \\
\hline & Allocation $A$ & 250,270 & 178,510 \\
\hline & Allocation B & 212,270 & 93,460 \\
\hline
\end{tabular}


Table 16. Simulation results for $V P_{0}: 4 \operatorname{DVAR}$ (1), Rebalancing case, $N=10,000$, $\alpha=5 \%$.

\begin{tabular}{|c|c|c|c|}
\hline \multirow{6}{*}{$g=1.4 \%$} & NO HEDGE & $E\left[V P_{0}\right]$ & $C V a R_{V P}(\alpha)$ \\
\hline & Allocation $A$ & $-92,000$ & $-1,102,900$ \\
\hline & Allocation $B$ & $-325,260$ & $-1,810,400$ \\
\hline & STATIC HEDGING & & \\
\hline & Allocation $A$ & 248,360 & 180,280 \\
\hline & Allocation $B$ & 245,900 & 136,170 \\
\hline \multirow{6}{*}{$g=1.5 \%$} & NO HEDGE & & \\
\hline & Allocation $A$ & $-117,420$ & $-1,143,000$ \\
\hline & Allocation B & $-359,750$ & $-1,885,300$ \\
\hline & STATIC HEDGING & & \\
\hline & Allocation $A$ & 244,540 & 177,300 \\
\hline & Allocation $B$ & 235,060 & 125,910 \\
\hline \multirow{6}{*}{$g=2 \%$} & NO HEDGE & & \\
\hline & Allocation $A$ & $-268,510$ & $-1,426,700$ \\
\hline & Allocation $B$ & $-516,010$ & $-2,129,600$ \\
\hline & STATIC HEDGING & & \\
\hline & Allocation $A$ & 219,270 & 152,010 \\
\hline & Allocation $B$ & 183,300 & 71,280 \\
\hline
\end{tabular}

Table 17. Simulation results for $V P_{0}: 4 \operatorname{DVAR}(1)$, No Rebalancing case, $N=10,000$, $\alpha=5 \%$.

\begin{tabular}{|c|c|c|c|}
\hline \multirow{6}{*}{$g=1.4 \%$} & NO HEDGE & $E\left[V P_{0}\right]$ & $C V a R_{V P}(\alpha)$ \\
\hline & Strategy $A$ & 69,000 & $-780,400$ \\
\hline & Strategy $B$ & $-188,760$ & $-1,394,800$ \\
\hline & STATIC HEDGING & & \\
\hline & Strategy $A$ & 263,810 & 206,290 \\
\hline & Strategy $B$ & 271,860 & 170,990 \\
\hline \multirow{6}{*}{$g=1.5 \%$} & NO HEDGE & & \\
\hline & Strategy $A$ & $-11,720$ & $-822,500$ \\
\hline & Strategy $B$ & $-243,890$ & $-1,460,400$ \\
\hline & STATIC HEDGING & & \\
\hline & Strategy $A$ & 262,860 & 204,600 \\
\hline & Strategy $B$ & 260,000 & 160,030 \\
\hline \multirow{6}{*}{$g=2 \%$} & NO HEDGE & & \\
\hline & Strategy $A$ & $-163,680$ & $-1,093,200$ \\
\hline & Strategy $B$ & $-384,370$ & $-1,693,400$ \\
\hline & STATIC HEDGING & & \\
\hline & Strategy $A$ & 243,740 & 184,000 \\
\hline & Strategy $B$ & 217,660 & 116,900 \\
\hline
\end{tabular}


holds for each considered case. Indeed Allocation B is more risky, therefore we get higher expected liabilities.

In particular, in the no hedge case, Allocation $B$ always leads to a loss. Regarding Allocation A, even if we get some positive values, they are lower than the initial sum of 180,000 euros.

Looking at static hedging, $E\left[V P_{0}\right]$ is always lower if allocation $\mathrm{B}$ is chosen, but we never get negative values. This worsening is due to the aforementioned higher cost of the hedging portfolio (Table 13).

Moving from the case of rebalancing the basket of assets to not rebalancing it, the results improve in each case. Referring to static hedging strategy, this is a consequence of the higher cost of the hedging portfolio (Table 13) due to a higher level of the underlying volatility in the rebalancing case. Moreover the higher volatility of the underlying allows for higher extreme returns and consequently higher liabilities. This effect is observable for the no hedging case as well.

Finally let us look at the results obtained by means of two-dimensional VAR (1) and four-dimensional VAR (1) models (2D VAR (1) and 4D VAR (1), respectively). For the static hedging case, 2D VAR (1) gives slightly higher values than $4 \mathrm{D}$ VAR both in case of rebalancing or not. Even if the cost of the hedging portfolio is lower in the $4 \mathrm{D}$ VAR (1) case, we can observe that in $2 \mathrm{D}$ the asset "volatilies" are averaged out in the estimators but in $4 \mathrm{D}$ they are estimated separately. This allows for more extreme returns in the 4D model and consequently higher liabilities. This effect is stronger when not hedging.

Looking at Conditional Value at Risk values, we observe the same behaviour as the expected values, of course. In particular, in the no hedge case the difference between the conditional values at risk and the expected values is very high. This is due to the higher risk associated with this choice. Moreover, for both the no hedge and the static hedge cases, we observe a worsening in terms of conditional values at risk, when changing from allocation A to the more risky allocation B.

\section{Concluding Remarks}

This paper studies, from the insurer point of view, a VA product offering GMDB and GMMB guarantees. In particular, the financial position of an insurer issuing a homogeneous portfolio of VAs offering those guarantees, is assessed. To this aim, the expected value and the conditional value at risk of this portfolio are quantified in a real world scenario. The results under a static hedging strategy are compared with those obtained when not hedging. Static hedging, as expected, gives better results.

Both in static hedging and no hedging cases, the portfolio value depends on many factors and we investigate some of them. Regarding the choices offered to the policyholders, we look at two different asset allocations and, as expected, the portfolio value decreases as the asset allocation becomes more risky. 
A crucial aspect is the choice of a model for the asset returns and the discounting rate. This choice impacts the valuation of the portfolio for risk management purposes.

VA guarantees are linked to more that one asset and the use of single-asset (univariate) models for VA pricing and risk management purposes, may lead to results that are not reliable. We show the results obtained by modelling jointly the financial variables involved by means of two kinds of VAR (1) models. We observe that using a four-dimensional VAR (1) model gives lower values of the portfolio than a two-dimensional VAR (1) model. In the first case, the asset returns on each fund are combined in a single time series while in the four-dimensional VAR (1) model, the asset returns on each fund are modelled separately. This structure adds more information in modelling the financial variables and the results could be more reliable. This provides useful information to the insurer concerned about risk management.

We also consider two different accumulations functions depending on whether the basket of assets is rebalanced or not. The rebalancing case gives lower values of the portfolio.

Following the analysis perfomed in this paper, further work could be done. This may include, for instance, the implementation of a dynamic hedging strategy and the use of stochastic mortality models.

\section{References}

[1] Ledlie, M.C., Corry, D.P., Finkelstein, G.S., Ritchie, A.J., Su, K. and Wilson, D.C.E. (2008) Variable Annuities. British Actuarial Journal, 14, 327-389. https://doi.org/10.1017/S1357321700001744

[2] Kalberer, T. and Ravindran, K. (2009) Variable Annuities. A Global Perspective. Risk Books.

[3] Bauer, D., Kling, A. and Russ, J. (2008) A Universal Pricing Framework for Guaranteed Minimum Benefits in Variable Annuities. Astin Bulletin, 38, 621-651. https://doi.org/10.1017/S0515036100015312

[4] Bacinello, A.R., Millossovich, P., Olivieri, A. and Pitacco, E. (2010) Variable Annuities: Risk Identification and Risk Assessment. CAREFIN Research Paper 14/2010, 1-48. https://ssrn.com/abstract=1799266

[5] Feng, R. and Volkmer, H.W. (2012) Analytical Calculation of Risk Measures for Variable Annuity Guaranteed Benefits. Insurance: Mathematics and Economics, 51, 636-648. https://doi.org/10.1016/j.insmatheco.2012.09.007

[6] Li, J.S.-H. and Ng, A.C.-Y. (2013) Pricing and Hedging Variable annuity guarantees with multiasset stochastic investment models. North American Actuarial Journal, 17, 41-62. https://doi.org/10.1080/10920277.2013.773240

[7] Zivot, E. and Wang, J. (2006) Vector Autoregressive Models for Multivariate Time Series. In: Modelling Financial Time Series with S-PLUS, Springer Science, 385-429.

[8] Hardy, M. (2003) Investment Guarantees: Modeling and Risk Management for Equity Linked Life Insurance. John Wiley \& Sons, Hoboken, NJ.

[9] Milevsky, M.A. and Posner, S.E. (1998) A Closed-Form Approximation for Valuing Basket Options. The journal of Derivatives, 5, 54-61. https://doi.org/10.3905/jod.1998.408005 